\title{
TECNOLOGIAS EDUCATIVAS GERONTOGERIÁTRICAS NAS DIFERENTES TEMÁTICAS DE SAÚDE: UMA REVISÃO INTEGRATIVA
}

\section{GERONTOGERIATRIC EDUCATIONAL TECHNOLOGIES IN DIFFERENT HEALTH THEMATICS: AN INTEGRATING REVIEW}

\section{TECNOLOGÍAS EDUCATIVAS GERONTO-GERIÁTRICAS EN LAS DIFERENTES TEMÁTICAS DE SALUD: UNA REVISIÓN INTEGRATIVA}

Embert Luan Correa Pereira ${ }^{1}$, Gabriel Zanin Sanguino ${ }^{2}$, Tamiris Sales Ronchi ${ }^{3}$, Giselle Fernanda Previato ${ }^{4}$, André Estevam Jaques ${ }^{5}$, Vanessa Denardi Antoniassi Baldissera ${ }^{6}$.

\section{RESUMO}

Objetivo: Analisar as tecnologias educativas em saúde desenvolvidas para a população idosa brasileira nas diferentes temáticas. Método: Revisão integrativa de literatura realizada nas bases de dados Lilacs, BdEnf, MEDLINE, Web of Science e Scopus, com os descritores: tecnologia educacional e saúde do idoso. A revisão foi norteada pela seguinte questão: "Quais tecnologias educativas em saúde têm sido utilizadas para a população idosa brasileira, nas diferentes temáticas?" Resultados: Foram selecionados sete artigos, e emergiram três categorias temáticas: 1) Tecnologias educacionais utilizadas na educação em saúde com idosos brasileiros; 2) Temáticas utilizadas nas tecnologias educacionais na educação em saúde com idosos brasileiros; 3 ) Impactos das tecnologias educacionais na educação em saúde com idosos brasileiros. Conclusão: Observou-se que a temática é minimamente abordada, demonstrado pelo baixo número de artigos encontrados. É importante que as tecnologias educativas sejam amplamente discutidas, visto que esse instrumento facilita a interação entre profissionais e usuários dos serviços de saúde.

Descritores: Saúde do idoso; Tecnologia educacional; Educação em saúde.

\section{ABSTRACT}

Objective: To analyze the health education technologies developed for the Brazilian elderly population in the different themes. Method: An integrative literature review carried out in Lilacs, BdEnf, MEDLINE, Web of Science and Scopus databases, with the descriptors: educational technology and elderly health. The review was guided by the following question: "What educational health technologies have been used for the Brazilian elderly population in the different themes?" Results: We selected seven articles, and three thematic categories emerged: 1) Educational technologies used in education in health with Brazilian elderly; 2) Themes used in educational technologies in health education with Brazilian elderly; 3 ) Impacts of educational technologies on health education with Brazilian elderly. Conclusion: We observed that this theme is slightly addressed due to the low number of articles found. It is important that educational technologies be widely discussed, since this instrument facilitates the interaction between professionals and health services users.

Descriptors: Elderly health; Educational technology; Health education.

\section{RESUMEN}

Objetivo: Analizar las tecnologías educativas en salud desarrolladas para la población anciana brasileña en las diferentes temáticas. Método: Revisión integrativa de literatura realizada en las bases de datos Lilacs, BdEnf, MEDLINE, Web of Science y Scopus, con los descriptores: tecnología educativa y salud del anciano. La revisión fue orientada por la siguiente pregunta: “¿Qué tecnologías educativas en salud se han utilizado para la población anciana brasileña en las diferentes temáticas?" Resultados: Seleccionamos siete artículos, y surgieron tres categorías temáticas: 1) Tecnologías educativas utilizadas en la educación en salud con ancianos brasileños; 2) Temáticas utilizadas en las tecnologías educativas en la educación en salud con ancianos brasileños; 3) Impactos de las tecnologías educativas en la educación en salud con ancianos brasileños. Conclusión: Se observó que la temática es mínimamente abordada, por el bajo número de artículos encontrados. Es importante que las tecnologías educativas sean ampliamente discutidas, ya que este instrumento facilita la interacción entre profesionales y usuarios de los servicios de salud. Descriptores: Salud del anciano; Tecnología educacional; Educación en salud.

${ }^{1}$ Graduado em Enfermagem pela Universidade Estadual de Maringá. ${ }^{2}$ Graduado em Enfermagem pela Universidade Estadual de Maringá. Mestrando pela Escola de Enfermagem de Ribeirão Preto da Universidade de São Paulo. ${ }^{3}$ Graduada em Enfermagem pela Universidade Estadual de Maringá. ${ }^{4}$ Graduada e Mestre em Enfermagem pela Universidade Estadual de Maringá. ${ }^{5}$ Enfermeiro. Doutor em ciências pela Escola de Enfermagem de Ribeirão Preto/USP. Professor adjunto do Departamento de Enfermagem da Universidade Estadual de Maringá. ${ }^{6}$ Enfermeira. Doutora em Ciências pela Universidade de São Paulo (USP). Professora adjunta do Departamento de Enfermagem e docente do Programa de Pós-Graduação em Enfermagem da Universidade Estadual de Maringá. ; Available in: DOI: 


\section{INTRODUÇÃO}

$O$ aumento da longevidade é um evento mundial $^{1}$ que vem ocorrendo tanto em países desenvolvidos como nos países em desenvolvimento como o Brasil. Entre 2001 e 2011, o número de idosos passou de 15,5 milhões para 23,5 milhões. 0 estrato idoso com 80 anos ou mais chegou a $1,7 \%$ da população em 2011, correspondendo a mais de 3 milhões de idosos e a expectativa é que essa faixa etária ainda esteja em crescimento nos próximos anos, estimando-se que, em 2025, o Brasil se torne a sexta maior população de idosos no mundo ${ }^{2}$.

$\mathrm{Na}$ área da saúde, essa rápida transição demográfica traz grandes desafios, pois é responsável pelo surgimento de novas demandas na área, resultando em maior e mais prolongado uso de serviços de saúde ${ }^{1}$. Para cuidar do idoso, as ações dos serviços de saúde incluem práticas de educação nessa área, cujo uso de tecnologia tem sido uma realidade ${ }^{3}$.

A tecnologia é um termo complexo que abrange o conhecimento técnico e científico, cuja classificação depende de seu conteúdo, natureza ou emprego, caracterizando-se como um processo que envolve inúmeros saberes, habilidades, técnicas, equipamentos, sujeitos, entre outros instrumentos, com o intuito de elevar a eficiência humana nos diferentes setores.

Na saúde, implica-se como um conjunto de ações que possuem como objetivo incrementar o cuidado e o tratamento. Compreende um processo que envolve ações e atitudes baseadas em conhecimento científico, técnico, pessoal, cultural, socioeconômico e político, favorecendo o cuidado integral por meio das práticas em saúde ${ }^{4}$, das quais as educativas fazem parte.

Nesse contexto, o profissional enfermeiro apresenta-se como um importante educador em saúde quanto aos cuidados que o processo de envelhecer traz consigo. Para esse intento, a tecnologia educativa surge como instrumento facilitador para o processo de ensinoaprendizagem, proporcionando o desenvolvimento de habilidades e mediando a construção de conhecimentos para o cuidado ${ }^{5}$.

Destaca-se que as práticas educativas possuem importância inegável para a promoção da saúde, utilizadas como veículo transformador de práticas e comportamentos coletivos e individuais e no desenvolvimento da autonomia e da qualidade de vida dos seres humanos ${ }^{6}$. É considerada um dos principais dispositivos para a viabilização da promoção da saúde, auxiliando no desenvolvimento da responsabilidade individual e na prevenção de doenças ${ }^{7}$.

Isso posto, os métodos tecnológicos e educacionais para educação em saúde, com objetivo de promover melhora da qualidade de vida da população idosa é um caminho que melhor precisa ser desvelado para oportunizar aos profissionais da saúde, sobretudo enfermeiros, conhecer as tecnologias educativas já existentes para os idosos e sinalizar caminhos de incremento e superação.

Diante desse cenário, o presente estudo teve como objetivo, analisar as tecnologias educativas em saúde desenvolvidas para a população idosa brasileira nas diferentes temáticas de saúde.

\section{MÉTODOS}

O método de pesquisa utilizado foi a Revisão Integrativa da Literatura, que pautou-se em seis etapas para sua elaboração: definição da questão de pesquisa, estabelecimento de critérios de inclusão e exclusão com a busca na literatura, definição das informações a serem extraídas dos estudos, avaliação dos estudos incluídos, interpretação dos resultados e síntese dos dados.

A questão que norteou o presente estudo foi: "Quais tecnologias educativas em saúde têm sido utilizadas para a população idosa brasileira nas diferentes temáticas?"

A busca foi realizada no período de junho a julho de 2017, a partir das bases de dados Literatura Latino-Americana e do Caribe em Ciências da Saúde (Lilacs), Base de dados de enfermagem (BdEnf), Sistema Online de Busca e Análise de Literatura Médica (MEDLINE), Web of Science e Scopus.

Os critérios para inclusão dos estudos foram: artigos científicos completos, realizados no Brasil, disponibilizados nos idiomas português, inglês ou espanhol, publicados até o período de coleta de dados meados do ano de 2017 voltados às pessoas com 60 anos de idade ou mais, de ambos os sexos, independente da temática em saúde.

Foram excluídos os artigos não primários, como os de opinião e as revisões e aqueles que, após a leitura na íntegra, não responderam ao objetivo desta revisão. É importante ressaltar que não houve recorte temporal para, de fato, levantar as publicações existentes sobre a temática. 
Para a realização da busca foram utilizadas combinações com os seguintes Descritores em Ciência da Saúde (DeCS) e Medical Subject Headings (Mesh): Tecnologia Educacional (Educational Technology); Saúde do Idoso (Health of the Elderly) combinados por meio do operador booleano "AND".

Foram encontrados seiscentos e vinte estudos, sendo: cinco na base Bdenf, seis no Lilacs, quatrocentos e dezoito no Medline, trinta e três no Web of Science e vinte no Scopus. Foram selecionados para análise, após leitura do título e resumo, doze estudos. Desses estudos, cinco foram descartados por se tratar de duplicidade.

O processo de seleção dos estudos está simplificado através do fluxograma preconizado pelo Transparent Reporting of Systematic Reviews and Meta-Analyses (PRISMA, 2009) do ano de 2009 (Figura 1) que apresenta os quatro passos para organização dos estudos: identificação, seleção, elegibilidade e inclusão.

Figura 1 - Fluxograma de seleção dos artigos. Maringá (PR), Brasil, 2017.

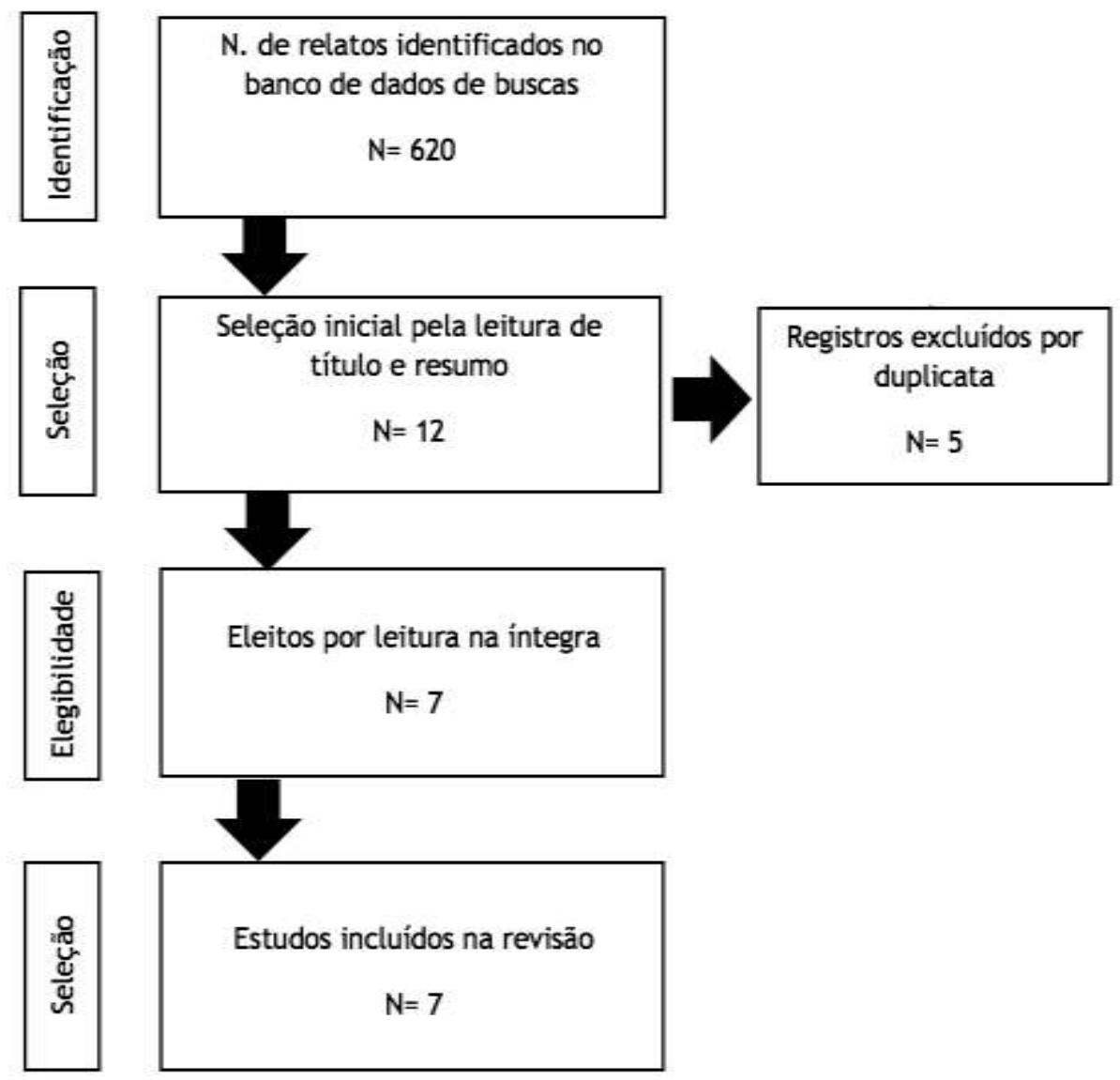

Fonte: Os autores.

Os estudos selecionados foram organizados em um quadro, contendo o perfil das publicações: título, autores, ano, tipo de estudo, objetivo e tecnologia encontrada. Os achados foram sistematizados em categorias temáticas e analisados na forma descritiva e interpretativa.

\section{RESULTADOS E DISCUSSÃO}

As características das publicações que compõe o estudo estão apresentadas (Figura 2) incluindo: nome do artigo, base de dados encontrada, autores, ano de publicação, tipo de estudo, objetivos, principais resultados e nível de evidência do estudo. 
Figura 2 - Características das sete publicações científicas brasileiras sobre as tecnologias educativas em saúde desenvolvidas para a população idosa brasileira nas diferentes temáticas de saúde. Maringá (PR), Brasil, julho de 2017.

\begin{tabular}{|c|c|c|c|c|c|}
\hline $\begin{array}{l}\text { Título do estudo/ } \\
\text { Base de dados }\end{array}$ & $\begin{array}{l}\text { Autor/ano de } \\
\text { publicação }\end{array}$ & $\begin{array}{l}\text { Tipo de } \\
\text { estudo }\end{array}$ & Objetivos & $\begin{array}{l}\text { Tipo de tecnologia } \\
\text { encontrada }\end{array}$ & $\begin{array}{l}\text { Nível de } \\
\text { evidência }\end{array}$ \\
\hline $\begin{array}{l}\text { Tecnologia } \\
\text { educacional como } \\
\text { estratégia de } \\
\text { empoderamento } \\
\text { de pessoas com } \\
\text { enfermidades } \\
\text { crônicas. } \\
\text { BDENF/LILACS. }\end{array}$ & $\begin{array}{l}\text { Berardinelli } \\
\text { LMM, Guedes } \\
\text { NAC, Ramos JP, } \\
\text { Silva NGM } \\
2014\end{array}$ & $\begin{array}{l}\text { Estudo } \\
\text { descritivo, de } \\
\text { abordagem } \\
\text { qualitativa. }\end{array}$ & $\begin{array}{l}\text { Desenvolver o trabalho em } \\
\text { grupo a fim de conhecer o } \\
\text { cotidiano e o processo } \\
\text { saúde/adoecimento/cuidado } \\
\text { das pessoas com } \\
\text { enfermidades crônicas; e } \\
\text { analisar se o trabalho de } \\
\text { grupo potencializou o } \\
\text { empoderamento e que } \\
\text { tecnologia educacional é } \\
\text { recomendada nas práticas } \\
\text { educativas para essas } \\
\text { pessoas. }\end{array}$ & $\begin{array}{l}\text { Diversas tecnologias } \\
\text { educacionais como a } \\
\text { dinâmica em grupo, } \\
\text { palestras, conversas e } \\
\text { panfletos informativos } \\
\text { voltadas ao } \\
\text { empoderamento. }\end{array}$ & 5 \\
\hline $\begin{array}{l}\text { Gerontotecnologia } \\
\text { educativa voltada } \\
\text { ao idoso } \\
\text { estomizado à luz } \\
\text { da complexidade. } \\
\text { BDENF/ MEDLINE/ } \\
\text { LILACS. }\end{array}$ & $\begin{array}{l}\text { Barros EJL, } \\
\text { Santos SSC, } \\
\text { Gomes GC, } \\
\text { Erdmann AL } \\
2012\end{array}$ & $\begin{array}{l}\text { Estudo } \\
\text { descritivo, de } \\
\text { abordagem } \\
\text { qualitativa. }\end{array}$ & $\begin{array}{l}\text { Apresentar a cartilha } \\
\text { educativa como um produto } \\
\text { geronto-tecnológico útil para } \\
\text { o cuidado ao idoso } \\
\text { estomizado à luz da } \\
\text { complexidade. }\end{array}$ & $\begin{array}{l}\text { Uma cartilha, que } \\
\text { apresentou-se como } \\
\text { uma geronto- } \\
\text { tecnologia capaz de } \\
\text { facilitar a compre- } \\
\text { ensão da pessoa idosa } \\
\text { esto-mizada e seu } \\
\text { familiar sobre os } \\
\text { direitos, conceitos e } \\
\text { tipos de estomas, } \\
\text { cuidados e impor- } \\
\text { tância da família e do } \\
\text { grupo de apoio para } \\
\text { cuidado. }\end{array}$ & 5 \\
\hline $\begin{array}{l}\text { Tecnologia } \\
\text { educacional } \\
\text { inovadora para o } \\
\text { empoderamento } \\
\text { junto a idosos com } \\
\text { diabetes mellitus. } \\
\text { LILACS/ SCOPUS. }\end{array}$ & $\begin{array}{l}\text { Hammerschmidt } \\
\text { KSA, Lenardt MH } \\
2010\end{array}$ & $\begin{array}{l}\text { Relato de } \\
\text { experiência. }\end{array}$ & $\begin{array}{l}\text { Refletir sobre o } \\
\text { empoderamento como } \\
\text { tecnologia educacional } \\
\text { inovativa para o cuidado de } \\
\text { si junto a idosos com } \\
\text { diabetes mellitus. }\end{array}$ & $\begin{array}{l}\text { O empoderamento } \\
\text { como tecnologia } \\
\text { educacional inova- } \\
\text { dora, que permite a } \\
\text { aprendizagem } \\
\text { dialógica e o } \\
\text { desenvolvimento de } \\
\text { consciência crítica na } \\
\text { qual o idoso com } \\
\text { diabetes encontra } \\
\text { sentido para um modo } \\
\text { de viver saudável } \\
\text { próprio/autônomo/ } \\
\text { personalizado. }\end{array}$ & 5 \\
\hline $\begin{array}{l}\text { Grupo aqui e } \\
\text { agora: uma } \\
\text { tecnologia leve de } \\
\text { ação sócio- } \\
\text { educativa de } \\
\text { enfermagem. } \\
\text { BDENF }\end{array}$ & $\begin{array}{l}\text { Lúcia Hisako } \\
\text { Takase } \\
\text { Gonçalves, } \\
\text { Jordelina Schier } \\
2005\end{array}$ & $\begin{array}{l}\text { Pesquisa } \\
\text { convergente- } \\
\text { assistencial. }\end{array}$ & $\begin{array}{l}\text { Desenvolvimento de uma } \\
\text { ação sócio-educativa de } \\
\text { enfermagem que } \\
\text { despertasse na pessoa idosa } \\
\text { e família, a percepção e a } \\
\text { compreensão do } \\
\text { autocuidado. }\end{array}$ & $\begin{array}{l}\text { Grupo de ação sócio } \\
\text { educativa, considerada } \\
\text { uma tecnologia leve de } \\
\text { cuidado, com foco na } \\
\text { educação para a saúde } \\
\text { aplicada pela } \\
\text { enfermagem, } \\
\text { desenvolvido junto aos } \\
\text { pacientes geriátricos } \\
\text { hospitalizados e seus } \\
\text { familiares } \\
\text { acompanhantes. }\end{array}$ & 4 \\
\hline
\end{tabular}




\begin{tabular}{|c|c|c|c|c|c|}
\hline $\begin{array}{l}\text { Título do estudo/ } \\
\text { Base de dados }\end{array}$ & $\begin{array}{l}\text { Autor/ano de } \\
\text { publicação }\end{array}$ & $\begin{array}{l}\text { Tipo de } \\
\text { estudo }\end{array}$ & Objetivos & $\begin{array}{l}\text { Tipo de tecnologia } \\
\text { encontrada }\end{array}$ & $\begin{array}{l}\text { Nível de } \\
\text { evidência }\end{array}$ \\
\hline $\begin{array}{l}\text { Contação de } \\
\text { história: } \\
\text { tecnologia } \\
\text { cuidativa na } \\
\text { educação } \\
\text { permanente para } \\
\text { o envelhecimento } \\
\text { ativo. } \\
\text { MEDLINE }\end{array}$ & $\begin{array}{l}\text { Nadia Pinheiro } \\
\text { da Costa, Sandra } \\
\text { Helena Isse } \\
\text { Polaro, } \\
\text { Eloá Aparecida } \\
\text { Caliari Vahl, } \\
\text { Lucia Hisako } \\
\text { Takase } \\
\text { Gonçalvesll } \\
2016\end{array}$ & $\begin{array}{l}\text { Pesquisa } \\
\text { Convergente } \\
\text { Assistencial. }\end{array}$ & $\begin{array}{l}\text { Avaliar a pertinência e } \\
\text { efetividade da tecnologia } \\
\text { "contação de histórias" } \\
\text { como estratégia cuidativo- } \\
\text { educacional no cultivo do } \\
\text { envelhecimento ativo pelos } \\
\text { usuários idosos de uma } \\
\text { Unidade Básica de Saúde no } \\
\text { município de Belém, do } \\
\text { Para. }\end{array}$ & $\begin{array}{l}\text { Uma tecnologia } \\
\text { cuidativo-educacional } \\
\text { de "contação de } \\
\text { histórias", como } \\
\text { estratégia inovadora } \\
\text { aplicável a serviços de } \\
\text { saúde, em matéria de } \\
\text { formação de } \\
\text { comportamentos que } \\
\text { conduzam ao cultivo } \\
\text { do envelhecimento } \\
\text { ativo e saudável. }\end{array}$ & 4 \\
\hline $\begin{array}{l}\text { Validação da } \\
\text { cartilha } \\
\text { informativa sobre } \\
\text { idosos } \\
\text { demenciados, } \\
\text { pelos enfermeiros } \\
\text { e acadêmicos de } \\
\text { enfermagem: } \\
\text { estudo } \\
\text { observacional- } \\
\text { transversal. } \\
\text { BDENF/ SCOPUS }\end{array}$ & $\begin{array}{l}\text { Alessandra } \\
\text { Conceição Leite } \\
\text { Funchal } \\
\text { Camacho, Louise } \\
\text { Theresa de } \\
\text { Araújo Abreu, } \\
\text { Bruna Silva Leite, } \\
\text { Ana Carolina de } \\
\text { Oliveira Mata, } \\
\text { Drielle dos } \\
\text { Santos Louredo, } \\
\text { Rafael Pires Silva } \\
2014\end{array}$ & $\begin{array}{l}\text { Pesquisa } \\
\text { quantitativa, } \\
\text { observacional } \\
\text { do tipo } \\
\text { transversal. }\end{array}$ & $\begin{array}{l}\text { Validar a cartilha informativa } \\
\text { como Tecnologia } \\
\text { Educacional (TE) sobre os } \\
\text { cuidados ao idoso com } \\
\text { demências pelos } \\
\text { enfermeiros e acadêmicos } \\
\text { de enfermagem. }\end{array}$ & $\begin{array}{l}\text { Uma cartilha voltada } \\
\text { para o cuidado a } \\
\text { pessoas com } \\
\text { demências, prevê- } \\
\text { nindo complicações, } \\
\text { desenvolvimento de } \\
\text { habilidades de seus } \\
\text { usuários e favorece a } \\
\text { autonomia e a } \\
\text { motivação da } \\
\text { enfermagem para } \\
\text { inventar novas } \\
\text { tecnologias } \\
\text { educacionais. }\end{array}$ & 5 \\
\hline $\begin{array}{l}\text { Cultivo do bem } \\
\text { viver de pessoas } \\
\text { idosas e tecnologia } \\
\text { cuidativo- } \\
\text { educacional de } \\
\text { enfermagem. } \\
\text { BDENF }\end{array}$ & $\begin{array}{l}\text { Thais Monteiro } \\
\text { Goes, Sandra } \\
\text { Helena Isse } \\
\text { Polaro, Lucia } \\
\text { Hisako Takase } \\
\text { Gonçalves } \\
2016\end{array}$ & $\begin{array}{l}\text { Pesquisa } \\
\text { convergente- } \\
\text { assistencial. }\end{array}$ & $\begin{array}{l}\text { Realizar avaliação } \\
\text { diagnóstica de condições de } \\
\text { vida e saúde dos idosos } \\
\text { convivendo em família e } \\
\text { comunidade, usuários de } \\
\text { uma Unidade Básica de } \\
\text { Saúde - UBS e testar o } \\
\text { desenvolvimento de uma } \\
\text { tecnologia cuidativo- } \\
\text { educacional. }\end{array}$ & $\begin{array}{l}\text { Uma tecnologia } \\
\text { cuidativo-educacional, } \\
\text { como uma tecnologia } \\
\text { leve de atos de relação } \\
\text { no cuidado. }\end{array}$ & 4 \\
\hline
\end{tabular}

Fonte: os autores.

Quanto às características dos artigos, todos se apresentaram na língua portuguesa. A data dos estudos se concentrou entre 2005 e 2016, destacando que a temática é recente e pouco difundida.

Ao analisar o conteúdo dos estudos selecionados, emergiram três categorias temáticas que foram foco das interpretações da revisão: 1) Tecnologias educacionais em saúde utilizadas com idosos brasileiros; 2) Temáticas utilizadas nas tecnologias educacionais na educação em saúde com idosos brasileiros; 3) Repercussões das tecnologias educacionais na educação em saúde com idosos brasileiros.

\section{1) Tecnologias educacionais em saúde utilizadas com idosos brasileiros}

Dentre as tecnologias educacionais para educar a população idosa do país, percebe-se a presença de dinâmicas em grupos ${ }^{9-10}$, rodas de conversas $^{11}$, palestras, oficinas e atividades individuais, caracterizadas como tecnologias leves ${ }^{10}$.

As tecnologias leves utilizadas como meios educativos, condensam em si, relações de interações de subjetividade entre trabalhador e usuário, de modo a facilitar o aprendizado. São geradoras de saúde quando voltadas ao cuidado, buscando reinventar seu saber e fazer. No caso do idoso, permite o acolhimento, formação de vínculos e fortalecimento de sua percepção e compreensão do autocuidado, sendo essencial à manutenção da autonomia e independência do viver com qualidade, conforme sua própria vontade ${ }^{9}$.

As dinâmicas em grupos e rodas de conversas são tecnologias leves que envolvem o processo de ensino-aprendizagem e que ocorrem de maneira compartilhada, proporcionando que os idosos socializem seus sentimentos, 
inquietações, relatos pessoais e, assim, partilhem, com o grupo, assuntos enriquecedores, a fim de potencializar condições de vida saudável e a autonomia. Com a presença de profissionais qualificados para a escuta ativa, o grupo se beneficia com conhecimentos de maneira prazerosa e satisfatória ${ }^{10}$.

Palestras, entretanto, que se opõe a essas estratégias mais horizontais de educação em saúde foram também encontradas e, também, situadas nas tecnologias leves, sinalizam a manutenção de práticas educativas hegemônicas que desprivilegiam e desrespeitam os saberes existentes, não favorecendo a inclusão social ${ }^{12}$.

Os materiais impressos, como cartilhas e folders, também foram utilizados como tecnologia educacional para idosos. É usual devido ao baixo custo de confecção e do alcance que o material pode atingir, contemplando um maior número de idosos, além da portabilidade da informação $0^{5,13}$.

A cartilha apresenta-se como uma gerontotecnologia capaz de facilitar a compreensão do idoso sobre diferentes temáticas de saúde ${ }^{14}$, sendo instrumento importante tanto na educação do indivíduo idoso, quanto de sua família ou outro grupo de apoio para cuidado ${ }^{(5,13)}$. Por outro lado, pode ser ferramenta inviável para idosos de baixa escolaridade, pela incompreensão do conteúdo do material, pois, mesmo que o analfabetismo geral venha diminuindo, ainda existe um significativo número de pessoas idosas que apresentam algum grau de dificuldade para a leitura ${ }^{15}$, sendo um ponto negativo de tais tecnologias impressas.

Não foram encontradas ferramentas digitais como tecnologias educacionais para idosos nos estudos revisados. No entanto, essas tecnologias podem colaborar com a qualidade de vida para essa população, pois esses se sentem inseridos no contexto social ${ }^{16}$.

2) Temáticas utilizadas nas tecnologias educacionais na educação em saúde com idosos brasileiros.

As principais temáticas educativas com idosos brasileiros foram sobre enfermidades crônicas não transmissíveis como fibromialgia, lúpus, hipertensão arterial sistêmica (HAS), Diabetes mellitus (DM), Parkinson, cardiopatias, fibromialgia associada à HAS e DM, e cuidados com idosos estomizados ${ }^{5,10,17}$.

Observa-se que as doenças crônicas não transmissíveis são foco da educação em saúde dos idosos, supostamente relacionadas ao perfil epidemiológico dessa população. Contudo, sinaliza um reducionismo à doença em detrimento à pessoa, ou seja, que a velhice é sinônimo de doença ${ }^{18}$, priorizando, nas práticas educativas, o idoso senil e não o senescente.

Embora as tecnologias educativas elencadas nesse estudo sejam centradas na doença, os seus benefícios não podem ser desconsiderados, pois asseguram, aos idosos, as mudanças necessárias na senilidade com foco na independência, na preservação cognitiva, autonomia $^{19}$, melhora na autoestima e autocuidado ${ }^{20-21}$.

\section{3) Repercussões das tecnologias educacionais na educação em saúde com idosos brasileiros.}

Uma das repercussões evidenciadas foi o empoderamento dos idosos, favorecendo a autonomia para o manejo de sua condição senil $^{5,22}$, sobretudo pela aprendizagem mediada por tecnologia, que torna os esclarecimentos claros e acessíveis ${ }^{9,19,23}$.

Outra repercussão foi a emancipação de saberes ${ }^{10}$ relacionada com questões de gênero, sexualidade e autocuidado, que fortaleceram o envelhecimento ativo ${ }^{5,22}$, resultando positivamente na qualidade de vida pelo manejo da capacidade funcional e domínio intelectual ${ }^{24}$.

Por fim, a satisfação dos idosos em participar de atividades educativas foi verificada $^{10,11}$, com destaque, para momentos de socialização $0^{10}$ e lazer cultural ${ }^{11}$, que mediaram essas abordagens.

A socialização no envelhecimento é desejável para evitar o isolamento social e colaborar com o desenvolvimento cognitivo, sendo essencial mantê-los assim ${ }^{18}$. Também o lazer edificante, presente nas atividades culturais, favorece a preservação cognitiva ${ }^{25}$. Ambas as atividades são descritas como preferências dos idosos, que mostram-se satisfeitos ${ }^{24}$.

\section{CONSIDERAÇÕES FINAIS}

Verificou-se que as principais tecnologias educativas utilizadas para idosos são classificadas como leves, a exemplo dos grupos, rodas de conversa, dinâmicas, palestras, cartilhas e panfletos. As doenças crônicas não transmissíveis foram a temática mais abordada, seguida de temas que envolvem o envelhecimento ativo. As principais repercussões envolveram o empoderamento, a emancipação de saberes e a 
satisfação na participação de momentos educativos.

Evidenciou-se, como limitação no estudo, a incipiência na produção de informações que envolvem o envelhecimento, a senescência e a abordagem dialógica da educação em saúde no entorno das tecnologias educativas, além do pequeno número de publicações sobre a temática que dificultou a síntese do conhecimento.

É importante que as tecnologias educativas gerontogeriátricas sejam amplamente discutidas no cenário da saúde, tanto entre os profissionais quanto entre a população idosa, e que novas tecnologias sejam desenvolvidas em prol da assistência aos indivíduos, como forma de inovação, capaz de produzir mudança no processo de envelhecer, principalmente com foco no empoderamento, autonomia e qualidade de vida nessa faixa etária.

\section{REFERÊNCIAS}

1- Veras R. É possível, no Brasil, envelhecer com saúde e qualidade de vida? Rev Bras Geriatr Gerontol. 2016;19(3):381-2. DOI: http://dx.doi.org/10.1590/1809-

98232016019.160100

2- Brasil. Instituto Brasileiro de Geografia e Estatística (IBGE). Síntese de indicadores sociais: Uma análise das condições de vida da população brasileira: 2016. Rio de Janeiro: IBGE; 2016.

3- Pilger C, Menon UM, Mathias TAF. Utilização de serviços de saúde por idosos vivendo na comunidade. Rev Esc Enferm USP 2013; 47(1): 213-20. DOI: 10.1590/S0080$\underline{62342013000100027}$

4- Carvalho AT, Oliveira MG. Tecnologias cuidativo- educacionais: Uma possibilidade para o empoderamento do/a enfermeiro/a? Rev Rene 2014;15(1):185-6. DOI: $\quad$ 10.15253/2175$\underline{6783.2014000100023}$

5- Barros EJL, Santos SSC, Gomes GC, Erdmann AL. Gerontotecnologia educativa voltada ao idoso estomizado à luz da complexidade. Rev Gaúcha Enferm. 2012;33(2):95-101. DOI: 10.1590/S198314472012000200014

6- Freire P. Pedagogia do oprimido. 62a ed. Rio de Janeiro: Paz e Terra; 2011.

7- Lopes MSV, Saraiva KRO, Ximenes LB. Analise do conceito de promoção da saúde. Texto Contexto-Enferm. 2010;19(3):461-8. DOI: 10.1590/S0104-07072010000300007

8- Mendes KDS, Silveira RCC, Galvão CM. Revisão integrativa: Método de pesquisa para a incorporação de evidências na saúde e na enfermagem. Texto Contexto-Enferm. 2008; 17(4):758-64. DOI: $10.1590 /$ SO104- $^{-}$ $\underline{07072008000400018}$

9- Gonçalves LHT, Schier J. "Grupo aqui e agora" Uma tecnologia leve de ação socioeducativa de enfermagem. Texto Contexto-Enferm. 2005; 14(2):271-9. DOI: 10.1590/S010407072005000200016

10- Berardinelli LMM, Guedes NAC, Ramos JP, Silva MGN. Tecnologia educacional como estratégia de empoderamento de pessoas com enfermidades crônicas. Rev Enferm UERJ 2014; 22(5):603-9. DOI: $10.12957 /$ reueri.2014.15509

11- Costa NP, Polaro SHI, Vahl EAC, Goncalves LHT. Storytelling: A care technology in continuing education for active ageing. Rev Bras Enferm. 2016;69(6):1068-75. DOI: 10.1590/0034-71672016-0390

12- Gonçalves GS, Sousa MNA, Medeiros VMM, Feitosa ANA. Práticas de humanização para o idoso na atenção primária à saúde. RIS 2016 [citado em 20 jun 2018]; 3(2):323-34. Disponível em:

http://interdisciplinaremsaude.com.br/Volume 1 0/Trabalho 06.pdf

13- Camacho ACLF, Abreu LTA, Leite BS, Mata ACO, Louredo DS, Silva RP. Validação de cartilha informativa sobre idoso demenciado pelos enfermeiros e acadêmicos de enfermagem: Estudo observacional-transversal. Rev Pesqui Cuid Fundam. 2014;6(1):8-16. DOI: 10.9789/21755361.2014.v6i1.8-16

14- Cordeiro LI, Lopes TO, Lira LEA, Feitoza SMS, Bessa MEP, Pereira MLD, et al. Validation of educational booklet for HIV/Aids prevention in older adults. Rev Bras Enferm. 2017;70(4):775-82. DOI: 10.1590/0034-7167-2017-0145

15- Arruda LM, Avansi TA. Analfabetismo na terceira idade: Pesquisa do analfabetismo em Sinop-MT. Even Pedagóg. 2014;5(2):435-42. DOI: 10.30681/2236-3165

16- Terra NL, Ferreira AJ, Tacques CO, Machado LR. Envelhecimento e suas múltiplas áreas do conhecimento. Porto Alegre: EDIPUCRS; 2016.

17- Hammerschmidt KSA, Lenardt MH. Tecnologia Educacional Inovadora para o empoderamento junto a idosos com diabetes mellitus. Texto Contexto-Enferm. 2010;19(2):358-65. DOI: 10.1590/S0104-07072010000200018

18- Amthauer C, Falk JW. A compreensão da velhice e do envelhecer na voz dos profissionais de saúde da família. Estud Interdiscipl Envelhec. 2014 [citado em 20 jul 2018]; 19(3):813-24. Disponível 
http://www.seer.ufrgs.br/RevEnvelhecer/article/ viewFile/47868/33287

19- Veras RP, Caldas CP, Cordeiro HA. Modelos de atenção à saúde do idoso: Repensando o sentido da prevenção. Physis 2013;23(4):1189-213. DOI: 10.1590/S0103-73312013000400009

20- Lima SAC, Parente ZR. Envelhecimento ativo: Um relato sobre o empoderamento do idoso inserido no Programa Universidade para Melhor Idade da Faculdade Leão Sampaio. Rev Interfaces 2016;3(11):59-62. DOI: 10.16891/2317-434X.366 21- Rêda RS, Rezende EJC, Monteiro NMM. Educação não formal e continuada: Uma contribuição do design para o empoderamento de idosos institucionalizados por meio de oficinas. In: Anais do 12을 Congresso Brasileiro de Pesquisa e Desenvolvimento em Design, 2016; Belo Horizonte. Belo Horizonte: UMA; 2016. p. 337583. DOI: $10.5151 /$ despro-ped2016-0290

22- Goes TM, Polaro SHI, Gonçalves LHT. Cultivo do bem viver das pessoas idosas e tecnologia cuidativo-educacional de Enfermagem. Enferm Foco 2016 [citado em 20 jun 2018]; 7(2):47-51. Disponível:

http://revista.cofen.gov.br/index.php/enfermage $\mathrm{m} /$ article/download/794/319

23- Cyrino RS, Silva LED, Souza MR, Borges CJ, Pereira LTS. Atividades lúdicas como estratégia de educação em saúde com idosos. Rev Ciênc Ext. 2016 [citado em 14 jun 2018]; 12(3):153-63. Disponível em:

http://ojs.unesp.br/index.php/revista proex/artic le/view/1324/1260

24- Wichmann FMA, Couto AN, Areosa SVC, Montañés MCM. Grupos de convivência como suporte ao idoso na melhoria da saúde. Rev Bras Geriatr Gerontol. 2013;16(4):821-32. DOI: 10.1590/S1809-98232013000400016

25- Gama TCCL, Amando AR, Macedo BCM, Silva GKS, Silva Júnior EX, Wannmacher CMD. Práticas educativas para o idoso: Um estudo comparativo. Rev Travessias 2016 [citado em 22 jun 2018]; 10(2):127-40. Disponível em: http://erevista.unioeste.br/index.php/travessias/ article/view/14660

Nota: Este artigo é fruto de um relatório de pesquisa produzido a partir de um projeto de iniciação científica (PIC), apresentado ao departamento de Enfermagem.

\author{
Endereço de correspondência: \\ Embert Luan Correa Pereira \\ Universidade Estadual de Maringá. Departamento de \\ Enfermagem - DEN/UEM. Avenida Colombo, 5.790 - \\ Campus Universitário - Bloco 01, sala 10 \\ CEP: 87020-900 - Maringá/PR - Brasil \\ E- mail: luan.embert@gmail.com
}

